\title{
Rechtsgeschichte
}

\section{Martin Otto}

Mehr individuelle Selbstbestimmung wagen! 


\section{Mehr individuelle Selbstbestimmung wagen!*}

Im Jahre 2004 rezensierte Martin Becker, Richter am Arbeitsgericht Frankfurt am Main, für die vom Deutschen Gewerkschaftsbund herausgegebene Zeitschrift »Arbeit und Recht « die Leipziger Dissertation von Annett Böhm, einer Stuttgarter Rechtsanwältin mit Schwerpunkt Arbeitsrecht. ${ }^{\mathrm{I}}$ Gegenstand der Dissertation war eine wissenschaftliche Biographie des Arbeitsrechtlers Arthur Nikisch, und Becker sparte nicht an Kritik, auch wenn er einzelnen Teilen das Prädikat »verdienstvoll« zusprach. Becker kritisierte den häufigen Gebrauch von Adjektiven wie »verdient « und »legendär «, wurde aber noch grundsätzlicher: Die Verfasserin habe »jeglichen Bezug zu den Gestaltungsfaktoren der sich durch die Epochen entwickelnden Arbeitsverfassung vermissen « lassen. Indem sich Becker selbst von Nikisch abgrenzte, formulierte er seine eigene arbeitsrechtliche »Gedankenwelt «. Nikisch habe sich »von Beginn seiner wissenschaftlichen Laufbahn an nicht im Zentrum einer Gedankenwelt der pluralistischen, freiheitlichen, gewaltenteilenden, rechtsstaatlichen Demokratie, der Autonomie und Selbstbestimmung im Arbeitsrecht und der Modernität insgesamt, sondern umgekehrt in einer Gedankenwelt der wachsenden Verherrlichung des Dienens, des Befehlsrechts, des Verfügungsrechts des AG [Arbeitgebers, M.O.] über den AN [Arbeitnehmer, M.O.], der Betriebsverbundenheit, des germanischen Gemeinschaftsverhältnisses «, also » unfreiheitlicher Chiffren «, befunden. Beckers Kritik blieb nicht unwidersprochen; andere Rezensenten zeichneten unter ausdrücklichem Verweis auf den »allein nach den moralischen Maßstäben der Zeitgeschichte « urteilenden Becker ein deutlich positiveres Bild. ${ }^{2}$
Wie Annett Böhm hatte auch Martin Becker mit einem Thema aus der Arbeitsrechtsgeschichte promoviert. ${ }^{3}$ Dieser Arbeit hat er jetzt das zweite Buch folgen lassen, eine unmittelbare Fortsetzung des ersten Buches. Nach »Arbeitsvertrag und Arbeitsverhältnis « im Kaiserreich geht es nun um diese Schlüsselbegriffe des Arbeitsrechts in Weimarer Republik und Nationalsozialismus. Zu Recht stellt Becker gleich eingangs »immer noch vorhandene Schwierigkeiten mit der Behandlung der Epochen der Weimarer Republik und des Nationalsozialismus « fest. Im Arbeitsrecht gebe es eine Neigung, rechtshistorische Methodik aus tages- oder rechtspolitischen Beweggründen zu instrumentalisieren: »Einzelfragen der heutigen Arbeitsrechtsdogmatik [...] werden herausgegriffen, auf literarische Äußerungen einzelner Autoren während einer bestimmten Epoche bezogen, um dann eine Kontinuität im Denken, eine Verwicklung, gar eine Mitschuld bestimmter Personen in nationalsozialistisches Unrecht $\mathrm{zu}$ behaupten oder $\mathrm{zu}$ widerlegen. ${ }^{4}$ So hatte der Konstanzer Emeritus Bernd Rüthers 1998 die Wurzeln des von ihm kritisierten Kündigungsschutzes im Nationalsozialismus und der dort propagierten Formel vom »Arbeitsverhältnis als Lebensbund « gesehen; dies glaubte er an einem 1939 erschienenen Aufsatz des katholischen Arbeitsrechtlers Wilhelm Herschel festmachen zu können, dem er ausdrücklich eine Mitschuld an der Arbeitslosigkeit I998 zuwies. Der Kölner Arbeitsrechtler Peter Hanau sah sich zu einer Verteidigung des I986 verstorbenen Herschel veranlasst.

Martin Becker versteht das Arbeitsverhältnis keineswegs als Lebensbund; entscheidend ist für

\footnotetext{
* Martin Becker, Arbeitsvertrag und Arbeitsverhältnis während der Weimarer Republik und des Nationalsozialismus (Juristische Abhandlungen 44), Frankfurt a. M.: Klostermann 2005 , XVI, 627 S., ISBN 3-465-03353-I; Seitenangaben ohne weiteren Nachweis beziehen sich auf dieses Werk.
}

I Martin Becker, Rezension von AnNett BöHm, Arthur Philipp Nikisch - Leben und Wirken, Berlin 2003, in: Arbeit und Recht 2004, 300-302.

2 ERwin Fromm, Rezension von Annett Böhm, Arthur Philipp Nikisch - Leben und Wirken, Berlin 2003, in: RdA 2005, 64 f. Auch auf die Arbeit von Annett Böhm kann hier nicht gebührend eingegangen werden; allerdings ist
Becker in seiner Kritik an dem Gebrauch von unbestimmten Eigenschaftswörtern und Wertungen (»einer der Großen des Arbeitsrechts « usw.) unbedingt zuzustimmen.

3 Martin Becker, Arbeitsvertrag und Arbeitsverhältnis in Deutschland. Vom Beginn der Industrialisierung bis zum Ende des Kaiserreichs, Frankfurt am Main I995.

4 Becker i i. 
ihn der »in den Art. I, 2 und I 2 GG enthaltene Messpunkt, nämlich individuelle Selbstbestimmung der am Arbeitsverhältnis beteiligten Arbeitgeber und Arbeitnehmer «. ${ }^{5}$ Gegenwärtig werde aber vielerorts die Verrechtlichung des Arbeitsrechts beklagt; »heute « sei »vornehmlich « von » Treue und Fürsorge oder von sonstigen Formelkompromissen im Arbeitsrecht die Rede«. Ähnlich hatte sich Becker bereits in seiner Rezension von 2004 geäußert; dort war vom »diffusen Gedanken der Betriebsverbundenheit « und »unbestimmten Treue- und Fürsorgepflichten " die Rede gewesen. ${ }^{6}$ Für Becker spricht gegen die Verwendung dieser Begriffe bereits »deren ideologische Belastung, die mangelnde richterliche Bindungswirkung, die hiermit verbundene Pflichtenlage im Arbeitsverhältnis «; zudem würden »diese Begriffsbildungen dem heutigen Arbeitsrecht, seiner verfassungsrechtlichen Veranlassung und Begründung, schließlich der individuellen Selbstbestimmung der am Arbeitsverhältnis beteiligten Arbeitgeber und Arbeitnehmer nicht gerecht «. Schon wegen der Grundrechtsgebundenheit des Arbeitsrechts dürfe das Arbeitsverhältnis »nicht mehr als Gewaltverhältnis einer Person über eine andere im Sinne einer Subjekt-Objekt-Beziehung bewertet werden ", denn das Arbeitsrecht sei trotz alledem »eine Rechtsbeziehung gleichgeordneter Vertragspartner ${ }^{7}{ }^{7}$ Die Aufgaben von Arbeitsrecht und Arbeitsrechtsgeschichte benennt Becker selbstverständlich. "Aufgabe des heutigen Arbeitsrechts ist es, sachlich nicht gebotene Abhängigkeiten zu beseitigen. ${ }^{8}$ Und die Arbeitsrechtsgeschichte habe »sich von einem narrativen Historismus $\mathrm{zu}$ verabschieden und die weitreichenden sozialen und politischen Veränderungen während der Weimarer Republik und des Nationalsozialismus als realgeschichtlichen Rahmen mit einzubeziehen ${ }^{9}{ }^{9}$
Becker beginnt mit der Weimarer Reichsverfassung vom II. August I9I9 und bezieht die Entstehungsgeschichte mit ein. Die Bedeutung der Verfassung für das Arbeitsrecht ist tatsächlich groß, als erste deutsche Verfassung besaß sie mit dem fünften Abschnitt ihres zweiten Hauptteils unter der Überschrift "Das Wirtschaftsleben « einen Normenkatalog, den man als sozialpolitisches Programm lesen konnte. Manche Verfassungsväter, so der sozialdemokratische Arbeitsrechtler Hugo Sinzheimer, wollten über den »Räteartikel « I 65 mit der »Idee der sozialen Selbstbestimmung « die politische Demokratie zu einer "sozialen Demokratie" entwickeln. Während der Verfassungsberatungen hatte Sinzheimer sogar gefordert, den grundrechtlichen Schutz des Einzelnen auch gegenüber »anderen sozialen Gewalten, die mitunter weit mächtiger als der Staat « seien, zu gewährleisten. ${ }^{\text {ㅇ }}$ Eine Drittwirkung von Grundrechten als anerkannte Rechtspraxis sollte es aber erst nach dem Zweiten Weltkrieg geben. Becker legt die unterschiedlichen Standpunkte, die sich auch in der Verfassungsinterpretation fortsetzten, überzeugend dar, beschränkt sich auf Wesentliches und referiert sachlich und ruhig. Seine Sympathie für "soziale Selbstbestimmung " verheimlicht er dabei nicht, lässt aber auch andere zeitgenössische Ansichten zu Wort kommen. Die meisten sozialdemokratischen Autoren hätten die Weimarer Verfassung »nicht als Erfüllung ihrer politischen Wünsche, sondern mehr als Weg zu ihrer Verwirklichung " gesehen, ${ }^{\mathrm{II}}$ ein pragmatischer Standpunkt, der an praktischen, auf Ausgleich bedachten Lösungen interessiert ist; man tut Martin Becker als Wissenschaftler und Richter sicher nicht unrecht, wenn man ihm Sympathien für diesen Standpunkt unterstellt.

Beckers weiteres Vorgehen nach einer umfangreichen Darstellung der Arbeitsverfassung

5 Becker I7.

6 BeCKer (Fn. I), 302. Ähnlich

dann: BECKER 254.

7 BECKER $29 \mathrm{f}$.

8 BeCKer 30.

9 BECKER 22.

io Hier zitiert nach Walter Pauly,

Grundrechtslaboratorium Wei-

mar, Tübingen 2004, 3.

오ํ

i I BeCKer 74.

Otto, Mehr individuelle Selbstbestimmung wagen! 
der Weimarer Republik, wobei seine knappe und prägnante Beschreibung der unterschiedlichen Gewerkschaften und die gelungene Zusammenfassung des »Ruhreisenstreits « hervorzuheben sind, ist enzyklopädisch. Anhand der Schriften von fünfzehn Arbeitsrechtlern werden die unterschiedlichen Auffassungen zu »Arbeitsvertrag und Arbeitsverhältnis in der Rechtslehre « dargestellt. Es finden sich die wichtigsten Vertreter an den Universitäten, Kaskel in Berlin, Richter und Jacobi in Leipzig, Molitor in Greifswald, Sinzheimer in Frankfurt, Kreller in Tübingen, Oertmann in Göttingen, Nikisch in Dresden, schließlich Hueck, Nipperdey und Herschel, die zu unterschiedlichen Zeiten hauptsächlich in Köln oder Jena tätig waren. Nicht zu den Universitätsjuristen ist der zeitweilig sehr einflussreiche, aus der Sozialreform kommende Beamte Heinz Potthoff zu rechnen, ferner der Ministerialbeamte Melsbach und der Richter am Bayerischen Obersten Landesgericht Wilhelm Silberschmidt.

Auch zur Weimarer Republik rechnet Becker den Austromarxisten Karl Renner. Warum der spätere österreichische Bundespräsident aufgeführt wurde, ist nicht klar ersichtlich. Zwar verstand sich die, in der Begrifflichkeit von Roland Dubischar, ${ }^{\mathbf{I 2}}$ scientific community der Arbeitsrechtswissenschaftler in der Weimarer Republik durchaus "großdeutsch «, und gerade Renner hatte dies immer wieder von einem linksnationalen Standpunkt mit Nachdruck betont. Auch eine arbeitsrechtliche Zusammenarbeit zwischen Wien und Berlin auf Ministerialebene gab es. Zum Arbeitsrecht der Weimarer Republik konnte Renner aber kaum beitragen. Auch Becker zitiert ausschließlich die I904 erschienene Schrift »Die Institution des Privatrechts und ihre soziale Funktion", also ein schon zeitlich nicht zur Weimarer Republik gehörendes Buch. Arbeitsrechtler, die unbestreitbar zur Weimarer Repub- lik gehörten, etwa Nawiasky, Hoeniger oder auch originelle Außenseiter wie Karl Korsch und Eugen Rosenstock-Huessy, ${ }^{13}$ sucht man dagegen vergeblich. An dem Verdienst Beckers, heute vergessene Autoren wie Silberschmidt wiederentdeckt zu haben, ändert dies freilich wenig.

Das Prüfschema, dem Becker die fünfzehn Juristen unterzieht, hat er am deutlichsten in seiner kritischen Würdigung von Nikisch hervorgehoben: Anders als dieser wünsche er sich eine »individuelle Persönlichkeit mit verfassungsrechtlichen Freiheitsgarantien, die sich selbstbestimmt in das Unternehmen einfügt $« .{ }^{\mathbf{I} 4}$ Das freundlichste Bild zeichnet Becker von Walter Kaskel, bei dem »immer wieder « der »Gedanke der Gleichordnung und der individuellen Selbstbestimmung im Arbeitsverhältnis « anklinge, "wobei er den arbeitsvertragsrechtlichen Bestimmungsgrund beibehielt und ohne Herrschaftsund Treue- und Fürsorgekonzepte auskommen konnte ${ }^{\mathbf{I} 5}$ Das positive Urteil über den heute nur Insidern bekannten Kaskel ist erfreulich, zumal der 1928 verstorbene Berliner Professor bis heute mit unglücklichen Etikettierungen wie " arbeitgeberfreundlich " versehen wird. ${ }^{\text {I6 }}$ Es ist ein großes Verdienst Beckers, den großen Praktiker und rechtspositivistischen Autor Walter Kaskel gebührend gewürdigt zu haben. Indem Becker allerdings »Gleichordnung " und »individuelle Selbstbestimmung « zu seiner Messlatte erhebt, um die Konzepte von Arbeitsvertrag und Arbeitsverhältnis zu überprüfen, begibt er sich zugleich in die Gefahr, die Arbeitsrechtswissenschaft der Weimarer Republik ex post zu bewerten. Die Argumentation von Becker ist dabei in sich logisch. Sein Bild von der Weimarer Verfassung ist positiv, er sieht in ihr "Selbstbestimmungspotentiale ${ }^{\mathbf{I 7}}$ und "sozialstaatliche Grundentscheidungen $« .{ }^{\mathbf{I} 8}$ Das sind Wertungen, zu denen man im Jahre 2005 gelangen kann, und
I 2 Roland Dubischar, Zur Entstehung der Arbeitsrechtswissenschaft als Scientific Community, in: RdA I990, 83. Gegen den Begriff (»überprüfungsbedürftig «) BECKER 25.

I3 Zu Rosenstock-Huessy aber BECKER 68, der ihn leider zum Heidelberger (richtig: Leipziger) Privatdozenten gemacht hat.

I4 BECKER 252.

I 5 BECKER I $75 \mathrm{f}$.
I6 Vgl. nur Uwe Wesel, Recht, Unrecht und Gerechtigkeit. Von der Weimarer Republik bis heute, München 2003, I7: "Aber es gab auch Professoren, die mehr die Interessen der Unternehmer vertraten. Walter Kaskel zum Beispiel in Berlin. «

I7 BECKER 254.

I 8 BECKER 252. 
es gibt gute Gründe dafür. Eine andere Frage ist freilich, ob man von den Autoren der zwanziger Jahre erwarten kann, dass sie die Weimarer Verfassung auch so verstanden haben, wie Becker sie heute versteht. Auch ein Sinzheimer wohlgesonnener Autor wie Gerhard Anschütz, dem Becker sicher mit Sympathie gegenübersteht, sah im »Räteartikel « I65 WRV »nur Gesetzgebungsprogramm, also Zukunftsrecht «. ${ }^{\mathbf{1 9}}$ Wenn Becker Art. I 65 interpretiert, nimmt er dabei teilweise einen von Franz Leopold Neumann und in abgeschwächter Form auch von Nipperdey vertretenen Standpunkt ein. ${ }^{20}$ Das Reichsgericht teilte diesen Standpunkt nicht. Nun ist das Reichsgericht weder sakrosankt noch unfehlbar, und für den Standpunkt von Becker, Nipperdey und Neumann spricht tatsächlich einiges. Doch ist es nicht unhistorisch, aus heutiger Sicht dem Reichsgericht vorzuwerfen, die WRV falsch interpretiert $\mathrm{zu}$ haben? In nächster Konsequenz wäre auch allen damaligen Autoren, die sich lege artis auf das Reichsgericht berufen hatten, ein abwegiger Standpunkt zu unterstellen. Die Arbeitsrechtsgeschichtsschreibung hört hier auf, man befindet sich bereits in der Rechtspolitik.

An anderer Stelle kritisiert Becker an Nikisch Unkenntnis der »Systemstrukturen « und "Selbstbestimmungspotentiale « der WRV, zugleich aber den »Vorrang des staatlichen Gesetzes. "2I Doch war nicht die WRV auch für Nikisch ein staatliches Gesetz? Der arbeitsrechtliche Standpunkt von Nikisch mag nicht behagen, doch von ihm zu verlangen, die WRV so zu lesen, wie wir es heute tun, über 70 Jahre nach ihrem faktischen Außerkrafttreten, das ist doch etwas zuviel verlangt. Dabei verteilt Becker seine Kritik keinesfalls einseitig. Ausgerechnet der revisionistische Sozialdemokrat Karl Renner sieht sich mit dem Vorwurf der »Refeudalisierung der Arbeitsverhältnisse in der liberalen Wirtschafts- und Arbeitsverfassung " konfrontiert; ${ }^{22}$ zudem übersehe der Marxist Renner bei seiner Kapitalismuskritik, dass es nicht immer der Eigentümer sei, »der die Geschicke des Unternehmens leitet «. Dabei beruft sich Becker auf das Mitbestimmungsurteil des Bundesverfassungsgerichts I979, also eine dem I950 verstorbenen Renner schon aus biologischen Gründen völlig unbekannte Entscheidung, aber fast gewinnt man den Eindruck, Renner hätte die Entscheidung kennen müssen. Den arbeitsrechtlichen Ansatz von Walter Kaskel lobt Becker als »modern, verfassungsorientiert und freiheitssichernd $«{ }^{23}$ Aber was ist der Maßstab für Beckers Moderne? Gerade im Bereich des Arbeits- und Sozialrechts enthält der Begriff zahlreiche Fußangeln. So hat etwa Rolf-Peter Sieferle darauf hingewiesen, dass aus der »Perspektive des ausgehenden I9. Jahrhunderts « die sich in Deutschland ausbildende und von Becker honorierte »Ausprägung des Sozialstaats nicht zwingend " als "modern « wahrgenommen wurde. »Es gab nicht wenige Stimmen aus dem In- und Ausland, die in der Bismarckschen Sozialpolitik den traditionellen Überrest einer ständisch-paternalistischen Vergangenheit sahen, der durch konsequente Liberalisierung hinweg zu modernisieren sei. « ${ }^{\mathbf{2 4}}$

An einer Stelle ist bei Becker sogar von einer »fortschrittlichen Position « des NS-Rechts die Rede, nämlich der Aufhebung der Trennung von öffentlichem und privatem Recht. ${ }^{25}$ Gibt es denn Fortschritt im Recht, und dann auch noch im NS-Recht? Die Kapitel zum Nationalsozialismus überzeugen gleichwohl. Nach einer prägnanten Einführung führt Becker nach seinem Schema dreizehn Einzelautoren und deren Standpunkte auf. Gegenüber Weimar neu hinzugekommen sind die Hochschullehrer Joerges, Siebert, Dersch und Dietz, ferner die aus der Verwaltung stammenden Autoren Müllereisert

I9 Gerhard Anschütz, Die Ver-

fassung des Deutschen Reichs, I4. Aufl. Berlin I933, 744.

20 BECKER $94 \mathrm{f}$.

2 I BECKER 254.

22 BECKER 216.

23 BECKER I74.

24 Rolf-Peter Sieferle, Die Kon-

servative Revolution, Frankfurt

a. M. I995, 201.

옹

25 BECKER 356. 
und Rhode. Den großen Aderlass, den der Nationalsozialismus für die deutsche Arbeitsrechtswissenschaft bedeutete, schildert Becker eindrucksvoll; Autoren wie Sinzheimer oder Jacobi durften in Deutschland nicht mehr publizieren und schwebten in Lebensgefahr. Die verbliebenen Autoren hatten dagegen relativ wenige Anpassungsschwierigkeiten, wenn sie bereits in Weimar ihr Arbeitsrecht auf Befehlen und Dienen konzipiert hatten. Weite Teile der nationalsozialistischen Arbeitsverfassung waren bereits in der Weimarer Republik angelegt und konnten beliebig mit Inhalten gefüllt werden.

Manchmal schießt Becker mit seiner Kritik aber über das Ziel hinaus. An Nikisch kritisiert er eine » militaristische Ausdrucksweise $« .{ }^{26}$ Aber hat Becker nicht selbst betont, dass man den realgeschichtlichen Rahmen einbeziehen muss? Und zu diesem realgeschichtlichen Rahmen gehört, dass der Bereich des Militärischen während der Weimarer Republik und davor in weiten Kreisen der Bevölkerung zumindest neutral, wenn nicht positiv konnotiert war, bis weit in die SPD hinein. Dass die Selbstverwaltungskörperschaften der Krankenkassen, in denen Sozialdemokraten im Kaiserreich erste Verwaltungserfahrungen sammeln konnten, stolz als »Unteroffiziersschulen der Sozialdemokratie « bezeichnet wurden, spricht eine deutliche Sprache, vielleicht deutlicher als die von Becker gerügten Begriffe »Befehlsrecht «, »Befehlsgewalt « oder »Unterordnungsverhältnis«. Ein Pazifist war Nikisch sicherlich nicht, ein militaristischer Jurist, der in Begriffen wie »Manneszucht « schwelgte, aber auch nicht. Dabei ist Beckers Kritik an Nikisch insgesamt einleuchtend und wird durch solch übertriebene Kritik an der Wortwahl in ihrer Wirkung eher gemindert.

Übertriebene Kritik verdient auch Martin Becker nicht. Sein Buch ist gut geschrieben und mit Gewinn zu lesen. Wie sein Vorbild Kaskel nimmt Becker einen ruhig-sachlichen Standpunkt ein. Seine Ergebnisse überzeugen, wo er schildert, wie es gewesen ist. Vieles konnte man in dieser Form so prägnant bisher nicht lesen. Er zollt der Sozialpolitik des Kaiserreichs seine Anerkennung und ist ein leidenschaftlicher Verfechter der "Selbstbestimmungspotentiale " der WRV. Pauschale Kritik, die man in arbeitsrechtsgeschichtlichen Werken der siebziger Jahre zuhauf lesen konnte, widerlegt Becker eindrucksvoll, so die immer wieder beschworene »verpasste Chance «, sich I9I9 nicht für eine Rätedemokratie entschieden zu haben. Indem Becker die in der Weimarer Republik liegenden Wurzeln vieler nationalsozialistischer Arbeitsrechtler betont, verwahrt er sich ohne erhobenen Zeigefinger gegen eine Verteidigungshistoriographie, die der Kritik an einem Standpunkt damit beizukommen meint, dieser sei schon vor 1933 vertreten worden. Auch in das andere Extrem, vermeintlich nationalsozialistische Wurzeln der bundesdeutschen Rechtsordnung aufzuzeigen, verfällt Becker nicht, im Gegenteil, er belegt, dass die Wurzeln oft weiter zurückreichen, dass deren Alter aber kein Qualitätsmerkmal an sich ist. Nicht immer kann man Becker folgen, wo er bewertet. Sein fester arbeitsrechtlicher Standpunkt, den man aus guten Gründen teilen kann, ermöglicht ihm eine klare Perspektive. Manchmal hat man aber den Eindruck, dass Becker zu sehr aus einer Nachkriegsperspektive bewertet, und auch Begriffe wie "Selbstbestimmung « oder »Gleichordnung « sind nicht gegen die Gefahr gefeit, zu unbestimmten Rechtskategorien zu werden. Was man sich gewünscht hätte, wären mehr biographische Details zu den behandelten Juristen. Waren die Vornamen und Lebensdaten einiger Autoren (Melsbach, Müllereisert, Mansfeld, Rhode) wirklich nicht feststellbar? Oder 
wäre das schon ein narrativer Rückfall gewesen? Am Schluss vermisst man etwas den Ausblick; in seinen Ergebnissen beschränkt sich Becker mehr oder weniger auf eine Zusammenfassung. Auf eine Fortsetzung in Form eines dritten Buches über Arbeitsvertrag und Arbeitsverhältnis in der Rechtslehre von Bundesrepublik Deutschland und DDR wartet man jedenfalls mit Spannung.

Martin Otto

\section{Monumente*}

Michael Kittner ist kein professioneller Rechtshistoriker. Er hat an der Universität Arbeits-, Sozial- und Wirtschaftsrecht gelehrt, vor allem aber war er über ein Vierteljahrhundert hinweg der Syndikus der Industriegewerkschaft Metall und somit der womöglich wichtigste juristische Kopf des Deutschen Gewerkschaftsbundes. In seine Amtszeit fiel die bis jetzt und, wie man mit Blick auf das heutige Deutschland meinen kann, für lange letzte hohe Konjunktur des Arbeitskampfes - mit spektakulären Streiks und Aussperrungen, die in den späten 7oer, besonders aber in den frühen 8oer Jahren des 20. Jahrhunderts die Öffentlichkeit in Atem hielten. Der Jurist Bernd Rüthers, als prominenter Schlichter (»Leber-Rüthers-Kompromiss « von I984) einer der bedeutenden Akteure jener Zeit, sprach im Zusammenhang mit der Expansion des industriellen Konfliktsystems von einem »Wirtschaftsbürgerkrieg « und traf mit dieser Übertreibung ganz gut die Stimmungslage in der Endzeit des rheinischen Kapitalismus. Heute ist das Regime des nationalstaatlich modulierten Fordismus erodiert, und mit ihm sind die Chancen dahingeschwunden, in den Arbeitsbeziehungen am großen Rad zu drehen. Die Berliner Republik ist nicht der Ort der arbeitspolitischen Emphase. Die Wasser der postnationalen Konstellation, die den Gewerkschaften, ihren Mitgliedern, aber auch so manchem Arbeitgeber heute wahrlich bis zum Halse reichen, legen anderes nahe als den Furor, der die alte - im Grunde genommen so überaus wirtschaftsfriedliche - Bonner Republik zu später Stunde noch einmal ergriffen hatte.

Wen es in solcher Lage nach Geschichte gelüstet, dem unterbreitet Michael Kittner ein erstaunliches, ein großartiges Angebot. Fast 800 Seiten zur Arbeitskampfgeschichte, aber anders als der Titel suggeriert nicht nur zu ihr, sondern $\mathrm{zu}$ den industriellen Beziehungen und zu deren Normativität überhaupt. Zum Organisationsrecht, zu den Tarifverträgen, zur Schlichtung, $\mathrm{zu}$ den industriellen Beziehungen und zu deren sozialpolitischer Umgebung: Alles, was die kollektive Welt der Arbeit ausmacht, ist in Kittners Buch erfasst, vermessen, bewertet und in Fasson gebracht.

Nach einer Ouvertüre zur vorkapitalistischen Zeit geht Kittner buchstäblich in die Vollen und stellt sich der ungeheuren Vielfalt der Sozialgeschichte und der noch ungeheureren Vielfalt der sozial-, rechts- und politikwissenschaftlichen Bemühungen um diese Geschichte auch. Von den Koalitionsverboten des früheren I9. Jahrhunderts bis zur Krise der Tarifautonomie und ihrer Annexinstitute in unseren Tagen das I9. und das 20. Jahrhundert begegnen uns

\footnotetext{
* Michael Kittner, Arbeitskampf.

Geschichte, Recht, Gegenwart, München: C. H. Beck 2005, XXIV, 783 S., ISBN 3-406-53580-I
} 